\title{
Interaction laser - métal en présence de plasmons de surface : vers une source de rayonnement UV et $X$
}

\author{
S. Bastiani, J. Kupersztych, P. Monchicourt et M. Raynaud \\ CEA, Service de Recherche sur les Surfaces et l'Irradiation de la Matière, DSMIDRECAM, \\ CEA Saclay, 91191 Gif-sur-Yvette, France
}

\begin{abstract}
Résumé : On présente une analyse expérimentale des électrons émis lors de l'interaction d'un laser ultra-bref avec des surfaces bi-couches $\mathrm{Au} / \mathrm{Al}$, où la résonance plasmon de surface du métal extérieur, excitée par le laser, excite elle-même une résonance à l'interface entre les deux métaux. On montre que l'émission, de durée quasi identique à celle de l'impulsion laser, augmente considérablement en présence de cette résonance. Un effet optimal est obtenu pour une épaisseur d'or de $40 \mathrm{~nm}$. Les électrons émis sont issus principalement de l'interface, et ont des énergies cinétiques pouvant atteindre plusieurs dizaines d'eV. En considérant que la traversée de la couche d'or, de l'interface à la surface, s'accompagne d'une émission de rayonnement de type bremsstrahlung, on dispose ainsi, grâce à cette résonance d'interface, d'une source ultra-brève et intense de rayonnement UV.
\end{abstract}

L'excitation de modes collectifs électroniques, en particulier des plasmons de surface, permet de rendre absorbante une surface métallique au rayonnement visible ou infra-rouge. Cette absorption se traduit par une émission électronique importante, résonnante avec l'excitation du plasmon de surface [1].

Cet effet peut être considérablement augmenté, via un nouveau schéma d'absorption du rayonnement, qui met en jeu un mécanisme collectif supplémentaire dans une structure métallique bi-couches [2]. Dans ce schéma, présenté sur la Figure 1, un plasmon de surface excité par laser à l'interface vide-métal 1 excite un autre mode collectif à la même fréquence à l'interface métal 1métal 2. Selon l'épaisseur du métal 1 , le champ électrique à l'interface peut atteindre une amplitude beaucoup plus grande que celle du plasmon de surface. On utilise ainsi cette nouvelle résonance, localisée à l'interface, comme moyen supplémentaire d'augmenter l'émission électronique pour la convertir ensuite en émission de rayonnement.

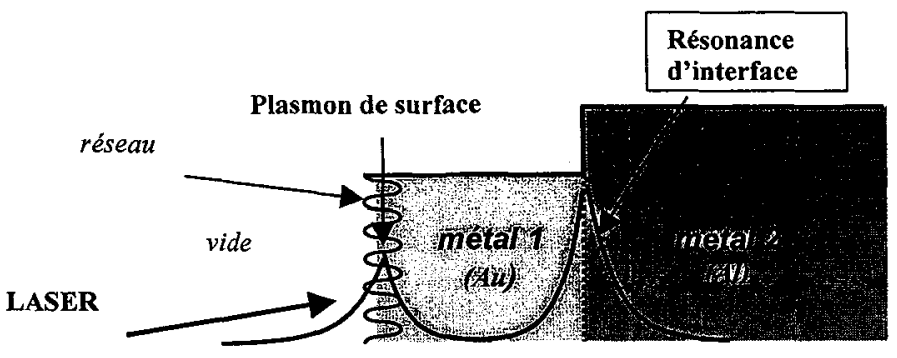

Figure 1: Description schématique de l'excitation par laser, via le plasmon de surface, d'un plasmon à l'interface entre deux métaux.

Une première série d'expériences a été réalisée avec le laser femtoseconde LUCA du DRECAM à Saclay (impulsions de $1 \mathrm{~mJ}$ à $800 \mathrm{~nm}$ en $80 \mathrm{fs}$ ), sur des surfaces périodiques bi-couches constituées 
de dépôts d'or de différentes épaisseurs déposés sur un substrat d'aluminium. L'or et l'aluminium ont été choisis afin de créer un important saut de densité d'électrons libres à l'interface entre les deux métaux. Les cibles utilisées sont des réseaux holographiques sinusoïdaux (ISA Jobin Yvon) ayant un pas de $150 \mathrm{t} / \mathrm{mm}$ et une profondeur de modulation de $50 \mathrm{~nm}$. Le choix de cibles ayant une surface périodique est nécessaire pour permettre le couplage entre l'onde electromagnétique du laser et le plasmon de surface.

La variation de l'émission électronique, en présence de la résonance, est reportée sur la Figure 2 pour des épaisseurs d'or allant de $5 \mathrm{~nm}$ (beaucoup plus petite que l'épaisseur de peau) à $200 \mathrm{~nm}$ (équivalente au métal semi-infini): un maximum d'émission est obtenu pour une épaisseur de l'ordre de $40 \mathrm{~nm}$, et l'émission correspondante est plus de 200 fois supérieure à celle provoquée par le seul plasmon de surface d'une couche d'or semi-infinie [3]. On en conclut donc que la résonance d'interface contribue de façon prépondérante à l'émission électronique.

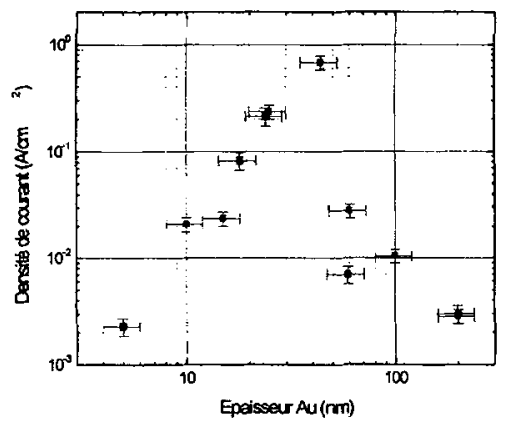

Figure 2: Variation de l'émission électronique en fonction de l'épaisseur d'or, pour une intensité laser de $1.6 \times 10^{8} \mathrm{~W} / \mathrm{cm}^{2}$

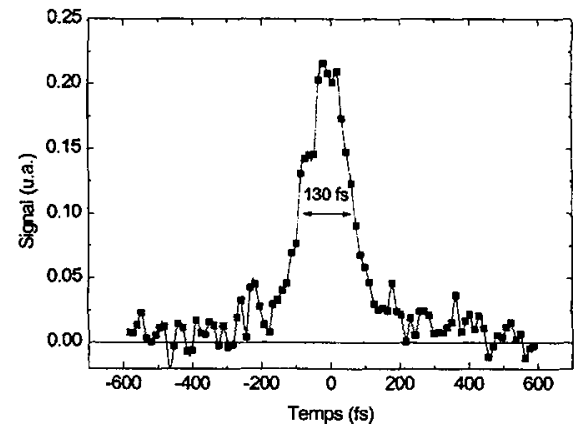

Figure 3: Signal électronique produit par deux impulsions laser de durée 80 fs et de même intensité, en fonction du retard entre les deux impulsions.

Une seconde propriété importante de l'émission électronique est sa durée. Mesurée par une technique d'autocorrélation [4] sur le signal électronique, cette durée est du même ordre que celle de l'impulsion laser, comme le montre la Figure 3. On dispose ainsi d'une source d'électrons qui est ultra-brève et synchrone avec l'impulsion laser.

Une troisième caractéristique de l'émission électronique est l'énergie cinétique des photoélectrons. Obtenue par spectrométrie à temps de vol, l'énergie de ces électrons, après traversée de la couche d'or, est d'autant plus grande que l'intensité laser est élevée. Elle varie de un à quelques dizaines d'eV dans le domaine exploré de $10^{8}$ à $10^{9} \mathrm{~W} / \mathrm{cm}^{2}$. On s'attend par conséquent à ce que cette traversée s'accompagne d'une émission de rayonnement, de type bremsstrahlung, dans le même domaine d'énergie.

Cet effet, qui sera testé très prochainement, constitue une voie nouvelle et intéressante pour la mise au point de sources UV ultra-brèves. Appliqué à des impulsions laser intenses générant des plasmas à la densité du solide, ce même effet devrait conduire à l'éjection d'électrons d'énergie beaucoup plus élevée et, par conséquent, à de nouvelles sources de rayonnement $\mathrm{X}$.

[1] J. Hofmann and W. Steinman, Phys. Stat. Sol. 30, K53 (1968); J.G. Endriz and W.E. Spicer, Phys. Rev. Lett. 24, 64 (1970); T. Tsang, T. Srinivasan-Rao and J. Fischer Optics Letters $\underline{15}$ 866 (1990); J.C. Gauthier et al, Phys. Plasma 4, 1811 (1997).

[2] J. Kupersztych and M. Raynaud, Solid State Com. 106, 785 (1998) et J. Phys.C6, 10669 (1994).

[3] S. Bastiani, J. Kupersztych, P. Monchicourt and M. Raynaud, à paraître.

[4] J.C. Diels and W. Rudolf, Ultrashort Laser Pulse Phenomena, Academic Press (1996) 